holmium by means of the double sodium sulfate separation. Accordingly, $5 \circ \mathrm{g}$. of samarium oxide were mixed with $25 \mathrm{~g}$. of holmium-yttrium oxides and this mixture subjected to a series of $3 \mathrm{I}$ fusions. The original holmiumyttrium oxides had an equivalent of 98.6 . The fractions gave the following absorptions and equivalents:

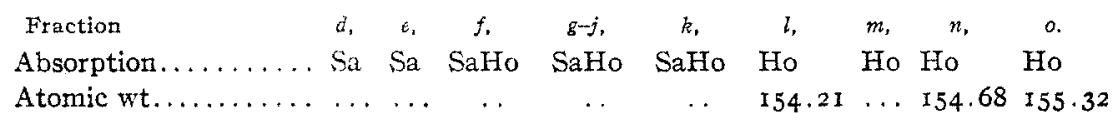

Most of the yttrium had concentrated toward the end showing the samarium lines. A very rapid concentration of holmium had taken place. This method was found to be the most efficient thus far tried. Work upon the separation of holmium from yttrium is being continued in this laboratory.

\title{
5. Summary.
}

I. The bromate method of fractionating the yttrium group earths for a more rapid concentration of dysprosium and holmium has been studied, and this method was found to be efficient for the concentration of holmium.

2. Some methods for the separation of holmium from yttrium were also studied. The fusion of the nitrates of a mixture of yttrium, holmium and samarium was found to give a very rapid concentration of holmium.

URBANA, ILLINOIS.

\section{OBSERVATIONS ON THE RARE EARTHS. VI. THE PURIFICA- TION AND ATOMIC WEIGHT OF DYSPROSIUM. ${ }^{1}$}

\author{
By H. C. KRRMERS, B. S. HoPKINS AND E. W. ENGLE.
}

Received November 30, 1917.

Contents.-I. Introduction. 2. History of Dysprosium. 3. Fractionation of the Bromates for the Purification of Dysprosium. 4. Fractionation of the Ethyl Sulfates for the Purification of Dysprosium. 5.. Preparation of Reagents Used in the Atomic-Weight Determinations. 6. Preparation of Dysprosium Oxide. 7. The Ratio of Dysprosium Sulfate to Dysprosium Oxide. 8. The Ratio of Dysprosium Oxide to Dysprosium Chloride. 9. The Ratio of Dysprosium Chloride to Silver. Io. Summary.

\section{Introduction.}

The object of the work described in this paper was to further purify dysprosium material already in a high state of purity. This purification was preliminary to a later object, namely, a further study of the atomic weight of dysprosium by the the use of the oxide-chloride ratio as used by Engle and Balke ${ }^{2}$ and by a comparative study of this ratio with other ratios.

1 Part of a thesis submitted by H. C. Kremers to the Graduate School of the University of Illinois as partial fulfilment of the requirements for the degree of Doctor of Philosophy.

This Journal, 39, 53 (I917). 


\section{History of Dysprosium.}

Our knowledge of the chemistry of dysprosium is very limited and but little concerning the element is found in the literature. In I 878 Delafontaine $^{1}$ announced a new element, philippia, giving a strong absorption band ( $=45 \mathrm{I} .5$ ). In $\mathrm{I} 880$ Delafontaine found that this band was the same as that described by Soret and Cleve for holmium. During the same year Soret ${ }^{2}$ announced that the philippia of Delafontaine and his holmium were the same and that the substance contained one body. In r 880 Lecoq de Boisbaudran ${ }^{3}$ by means of several hundred fractionations with ammonia and potassium sulfate succeeded in separating the supposed holmium of Soret into two elements. The element giving the absorption maxima: $=753,475,45$ ז.5 and 429.5 was called dysprosium.

It was not until I 906 that Urbain ${ }^{4}$ obtained some 50 grams of dysprosium oxide by fractionation of the ethyl sulfates and nitrates. An extensive study of the absorption spectra of the element was also made by the same author. The present accepted value of the atomic weight of dysprosium was determined by Urbain and Demenitraux. ${ }^{5}$ According to these authors the ratio which gave the most concordant results was the transformation of the octohydrated sulfate $\mathrm{Dy}_{2}\left(\mathrm{SO}_{4}\right)_{3} .8 \mathrm{H}_{2} \mathrm{O}$, into the oxide $\mathrm{Dy}_{2} \mathrm{O}_{3}$ by ignition at a white heat. As a mean of 12 determinations the value 162.54 was obtained as the atomic weight. Their greatest variation was between I62.29 and I62.75. Some fifteen compounds of dysprosium have been studied by Bourion, ${ }^{6}$ Urbain and Jantsch ${ }^{7}$ and Jantsch and Ohl. ${ }^{8}$ The atomic number of dysprosium, according to the table arranged by Mosley, ${ }^{9}$ is 67 , placing it between holmium 66, and erbium 68. Dushman ${ }^{10}$ states that the order of the atomic numbers of dysprosium and holmium in Mosley's table is apparently the reverse of that of the atomic weights. Harkins and Hall ${ }^{11}$ assign 66 as the atomic number of the element. Durrant ${ }^{12}$ also assigns the same number to dysprosium.

3. Fractionation of the Bromates for the Purification of Dysprosium.

In the fractional crystallization of the yttrium group bromates, by Engle and Balke, ${ }^{13}$ dysprosium bromate was obtained containing one or two per

1 Compt. rend., 87, 559 ( 1878 ).

${ }^{2}$ Ibid., 91,378 (1880).

${ }^{3}$ Ibid., 102, 1003 (1886); Chem. News, 53, 265 (1886).

4 Ibid., r42, 785 (1906).

'Ibid., I43, 598 (1906).

- Compt. rend., 145, 243 (1907); Ann. chim. phys., [8] 2I, 77 (1910).

7 Ibid., 146, 127 (1908).

8 Ber., 44, I274 (I9II).

- Phil. Mag., 27, 703 (1914).

10 Gen. Elec. Rev., 18, 614 (1915).

11 This Journal, 38, 2 (IgI6).

12 Ibid., 39, 62I (1917).

${ }^{18}$ Loc. cit. 
cent. of holmium, small amounts of neodymium, praseodymium and traces of terbium. This series was further fractionated as the bromates, during which time fractions were removed from the insoluble end as the neodymium and praseodymium collected here. As the holmium concentrated toward the soluble end these fractions were removed from time to time. These bromates were run through 125 series of recrystallizations. A careful spectroscopic examination indicated faint traces of neodymium and praseodymium in the least soluble fractions. The color of this oxide was slightly buff. Holmium was found in all of the fractions being more concentrated toward the soluble end. Since the amount of holmium present was estimated to change the atomic weight of the dysprosium no more than two or three units in the second decimal place it seemed that this material was sufficiently pure for an accurate determination of the atomic weight. Accordingly, Samples $;$ and 8 ('Table of Results) were selected from this series.

The bromate fractionation was not wholly ideal in the final purification of dysprosium; neodymium and praseodymium concentrated slowly toward the less soluble end; and the last traces of holmium were not removed by this method.

\section{Fractionation of the Ethyl Sulfates for the Purification of Dysprosium.}

It seemed highly desirable that the two ethyl sulfate series, which had been fractionated by Engle and Balke ${ }^{1}$ for the separation of dysprosium, be further fractionated with the hope that the last impurities might be removed. It was found that most of the fractions of the two above mentioned series had hydrolyzed during the warm summer months, and the majority of the fractions had to be recovered. The individual fractions were precipitated with oxalic acid, ignited to the oxide and weighed. The calculated amount of ethyl sulfuric acid was added to each oxide and agitated until complete solution had taken place. Absolute alcohol was used as the solvent in the fractionation of these series and the crystallization was allowed to take place in a refrigerator in which the temperature was $9^{\circ}$. By this method a temperature of $30-40^{\circ}$ was sufficient to dissolve the crystals and by keeping the fractions at this low and constant temperature during crystallization very little hydrolysis took place.

The first ethyl sulfate series, as left by Engle and Balke contained a fraction of one per cent. of terbium with slight amounts of neodymium and praseodymium toward the less soluble end. Holmium was found in all the fractions. This series was fractionated 65 times. Very nearly all of the neodymium and praseodymium had been removed during this time. A small amount of terbium was still present. The holmium could not be removed by this method.

1 Loc. cit. 
The second ethyl sulfate series contained besides the dysprosium very small amounts of terbium. This series was fractionated in the same manner as the first ethyl sulfate series with the hope that the last traces of terbium might be removed. After 60 recrystallizations it was found that some terbium still remained although a considerable amount had concentrated toward the insoluble end.

A comparison of the bromate and ethyl sulfate methods of fractional crystallization shows that the ethyl sulfates were more efficient for the removal of terbium, neodymium and praseodymium from dysprosium. Neodymium, praseodymium and terbium were removed only very slowly by the bromate method. By neither of the methods could all of the holmium be removed although the bromates were the best suited for this purpose.

\section{Preparation of Reagents Used in the Atomic-Weight Deter- minations.}

Water.-The ordinary distilled water was redistilled, after the addition of alkaline permanganate, from the still used in this laboratory for the preparation of conductivity water. The water thus prepared gave no test for chlorine by the most delicate nephelometric test.

Nitric Acid.-c. P. nitric acid was redistilled from quartz apparatus. The middle third was collected in a quartz flask and reserved for use. The acid thus prepared gave no test for chlorine.

Oxalic Acid.-C. P. oxalic acid was dissolved in hot water, filtered and recrystallized twice from conductivity water, containing ro\% redistilled nitric acid and lastly from conductivity water. Each yield was drained in a porcelain bowl centrifuge.

Hydrochloric Acid.-Concentrated C. P. hydrochloric acid was heated in a quartz distilling flask and the vapors passed over conductivity water in a quartz flask.

Hydrogen Chloride.-This was prepared by dropping concentrated C. P. sulfuric acid into a concentrated solution of C. P. hydrochloric acid. The gas was dried by passing through two towers, one meter high and $2.5 \mathrm{~cm}$. in diameter, filled with glass beads which were kept moist with conc. C. P. sulfuric acid. The entire apparatus was of glass and was continuous.

Nitrogen.-Air was saturated with ammonia, by bubbling through a wash bottle containing concentrated ammonium hydroxide solution, and passed through a quartz tube filled with copper gauze, heated to redness by means of an electric furnace. This nitrogen was bubbled through dil. sulfuric acid to remove the excess of ammonia. The gas was purified by passing through a train composed of vertical towers $60 \mathrm{~cm}$. high and $2.5 \mathrm{~cm}$. in diameter. The first tower contained solid glass beads moistened with dil. sulfuric acid. The second tower contained glass beads moistened with $5 \%$ silver nitrate solution. The third tower contained glass beads 
moistened with c. P. conc. sulfuric acid. The fourth tower contained solid sticks of potassium hydroxide. The fifth tower again contained glass beads and conc. sulfuric acid; and lastly the gass passed through a tube containing glass wool interspersed with freshly sublimed phosphorus pentoxide.

Air.-Air was purified in the same manner as was the nitrogen.

The purifying trains for hydrogen chloride, nitrogen and air were all mounted in a compact form on a wooden frame. All connections were made of glass or ground-glass joints. This apparatus was also used by Engle and Balke in their investigation on the atomic weight of dysprosium.

Silver.-The silver used in this investigation was prepared in essentially the same manner as that used by Richards and Wells ${ }^{2}$ in their investigation on the atomic weights of sodium and chlorine.

Thirteen hundred g. of C. P. silver bromide were reduced by a dry fusion, composed of the following charge: 8 parts sodium carbonate, 8 parts potassium carbonate, 3 parts of silver bromide and I part of flour. This mixture was fused in fire clay crucibles in a large gas-fired pot furnace. The melt was poured into conical iron molds, the silver buttons collecting in the bottom. These silver buttons were cleaned, etched with nitric acid, washed and dissolved in nitric acid. The silver nitrate was recrystallized 3 times from redistilled nitric acid and conductivity water. The crystals were drained in each case by means of a high-speed centrifuge. The silver nitrate solution was next reduced with freshly prepared ammonium formate and washed several times by decantation. The silver placed in large porcelain crucibles lined with pure carbon ${ }^{3}$ was fused in an electric furnace. The silver buttons were carefully cleaned, etched with nitric acid, washed, dissolved in redistilled nitric acid and again reduced with ammonium formate. The reduced silver was washed by decantation with conductivity water and fused in large porcelain crucibles lined with pure lime ${ }^{3}$ in an electric furnace. The silver thus obtained was carefully cleaned with a stiff brush, etched, washed and electrolyzed, using a bar of pure silver ${ }^{4}$ as the cathode. The fused silver was used as the anode and silver nitrate, made by dissolving some of the fused silver in nitric acid, formed the electrolyte. By carefully regulating the strength of the current a well crystallized deposit of silver was obtained. This electrolytic silver was well washed with conductivity water, dried and fused in large unglazed porcelain boats lined with pure lime. This final fusion was carried out in a porcelain tube electric furnace itn an atmosphere of pure hydrogen, prepared from zinc and hydrochloric acid and purified by passing through wash towers containing alkaline permanganate, silver sulfate, solid potas-

\footnotetext{
Loc. cit.

${ }^{2}$ Pub. Carnegie Inst., 28 (1905).

${ }^{3}$ Richards and Wells, Ibid.

- Sears and Balke, This Journal, 36, 833 (1915).
} 
sium hydroxide and lastly through a quartz tube containing heated platinized quartz, and a tube of glass wool interspersed with freshly sublimed phosphoric anhydride. Bars of silver weighing some $50 \mathrm{~g}$. each were obtained and were carefully cleaned, etched, washed and dried and preserved in a desiccator over solid potassium hydroxide. Before weighing the samples for analysis, these bars were cut into pieces, etched to the desired weight and washed with conductivity water, absolute alcohol and ether and heated to $120^{\circ}$ for some minutes.

All vessels used in the preparation of the reagents, described above and in the work following, were of platinum, quartz, or Jena and Non Sol resistance glass.

\section{Preparation of Dysprosium Oxide.}

The dysprosium oxide for the analysis was prepared as follows: The alcoholic solution of the ethyl sulfates was diluted with water, a few cc. of dil. sulfuric acid added and heated to boiling. All the barium, from the small amounts of barium ethyl sulfate present in the series, was removed as barium sulfate. The dysprosium was then precipitated with oxalic acid. The fractions from the bromate series were first precipitated with ammonia, washed, dissolved in nitric acid and from this point on the purification was the same as that of the material from the ethyl sulfate series. Several alternate precipitations with ammonia and oxalic acid were given to each sample. The ammonia precipitation was carried out by passing ammonia gas over the nitrate solution diluted to one liter and agitated until precipitation was complete. The hydroxides were washed by decantation. When washing was complete the hydroxides were dissolved in nitric acid diluted to one liter and heated to boiling. A hot solution of dil. oxalic acid was added slowly up to the point when precipitation began. By allowing the solution to cool a crystalline precipitate was obtained which was easily washed. After washing the oxalate was dried in an electric oven and ignited in platinum if another ammonia precipitation was desired. Each sample was precipitated 3 or more times with ammonia and usually the same number of times with oxalic acid, the last precipitation always being as the oxalate. The last precipitation' in each case was carried out by the use of redistilled nitric acid and conductivity water. The final oxalate was ignited for several hours in platinum in an electric muffle furnace at a temperature of $800^{\circ}$ and lastly for an hour at $900^{\circ}$.

\section{The Ratio of Dysprosium Sulfate to Dysprosium Oxide.}

The ratio mentioned above was used by Urbain and Demenitraux ${ }^{1}$ upon whose work the present accepted value is based. A sample of dysprosium was prepared by Engle and Balke in the same manner as prepared by the authors mentioned above. This in brief was as follows: The

${ }^{1}$ Compt. rend., 142, 785 (1906). 
concentrated sulfate solution was precipitated with a large excess of alcohol. This sulfate was washed with absolute alcohol, dissolved in water and crystallized on a steam bath. The sulfate was dried over sulfuric acid in a desiccator. Urbain and Demenitraux claimed that the pure octohydrated sulfate $\mathrm{Dy}_{2}\left(\mathrm{SO}_{4}\right)_{3} .8 \mathrm{H}_{2} \mathrm{O}$ was obtained in this way.

An alundum crucible was ignited to constant weight in a platinum wound muffle furnace at a temperature of $I 200^{\circ}$, and I. $33843 \mathrm{~g}$. of the sulfate, which had been dried over sulfuric acid for 12 months, was weighed out. This was ignited to constant weight, giving $0.66955 \mathrm{~g}$. as the weight of the oxide. This indicates an atomic weight of 173.291 . From this determination it would seem that a partial dehydration of the sulfate had taken place in the desiccator. Hopkins and Balke ${ }^{1}$ in their work on the atomic weight of yttrium found that the hydrated yttrium salts were not stable when kept over sulfuric acid, calcium chloride or partially dehydrated yttrium salts. The investigation of the dysprosium sulfate was not carried further since it did not appear that a hydrated sulfate of constant composition could be obtained under the present conditions.

\section{The Ratio of Dysprosium Oxide to Dysprosium Chloride.}

The method of procedure used here was essentially the same as that used by Egan and Balke in their study of a similar ratio for yttrium. Practically the same apparatus used in this work was used by Engle and Balke ${ }^{3}$ in their investigation on the atomic weight of dysprosium. The oxide while still hot from the ignition was transferred to the $25 \mathrm{cc}$. quartz reaction flask, the caps quickly adjusted on the inlet and outlet tubes and the flask cooled in a desiccator. The flask was hung in the balance case for 3 hours or more and weighed. Just before weighing one of the caps was loosened for a moment to equalize the pressure. The oxide was dissolved by addition of hydrochloric acid and gentle warming. No effervescence or spattering was noticed. The quartz reaction flask was then attached to the purifying train by 2 ground glass joints, one serving as an inlet and the other as an outlet for the gases. The purifying train was so constructed that air, nitrogen, hydrogen chloride or a mixture of any two or all of the gases could be passed into the flask at will. The procedure from this point on was varied somewhat from that used by Engle and Balke. An especially constructed oven made of asbestos wood 0.25 inch thick, was used to heat the flask. The inside dimensions were $4 \times 6 \times 7$ inches and mica windows were placed in two opposite sides. Coils of nichrome resistance wire served as the heating unit and by means of outside resistance any constant temperature up to $360^{\circ}$ could be maintained. This oven could be placed around the flask after it was attached to the train,

1 'ThIs JoUrnal, 38, 2332 (I9I6).

Ibid., 35, 365 (1913).

${ }^{3}$ Loc. cit. 
in such a manner that the entire flask together with the inlet and outlet tubes was inclosed and entirely protected from the laboratory gases. The various stages of the dehydration could be observed through the mica windows without disturbing the flask. Air was first passed through the flask while the temperature was IIO-II $5^{\circ}$. When the salt began to crystallize hydrogen chloride was mixed with the air until the salt had completely crystallized and dehydration had begun to take place. The air was now stopped and only hydrogen chloride passed through. At this point Engle and Balke passed nitrogen through the flask together with the hydrogen chloride. It was found that nitrogen could be omitted without any difficulty. As the dehydration took place the temperature was gradually raised to $125^{-1} 30^{\circ}$ and kept at this point until water ceased to escape. The temperature was raised to $200^{\circ}$, at which the last molecule of water of crystallization began to come off, the dehydration becoming complete at $230^{\circ}$. From this point on the temperature was gradually raised to $350^{\circ}$ and the oven then replaced by a small muffle furnace. When the temperature had reached a dull red the current of hydrogen chloride was stopped and the chloride fused with the Bunsen flame. In the last 4 determinations the chloride was fused by means of the electric furnace. After the flask had cooled somewhat the hydrogen chloride was replaced by dry air. With the exception of Samples I and 2 the exit air, from the displacement of the hydrogen chloride, was finally bubbled through silver nitrate solution until no further test for hydrogen chloride could be obtained. This insured the absence of all hydrogen chloride in the flask. When the flask had cooled to room temperature the outlet tube was first disconnected from the train and the cap adjusted. While the air was still passing the inlet tube was disconnected from the train and the cap quickly adjusted thus preventing the entrance of any moist air. The flask was hung in the balance case for several hours and weighed.

The dysprosium chloride formed in this manner dissolved completely in cold water to a clear solution. The dysprosium chloride solution from a preliminary run gave the following reactions toward several indicators: The solution was basic toward rosalic acid, basic to methyl orange, slightly acidic to congo red, slightly basic to cochineal, neutral to litmus and slightly acidic toward methyl red. These reactions indicated that the hydrogen-ion concentration of the solution ${ }^{1}$ was $10^{-5}$ or 6 .

Nine consecutive determinations were made. Samples I and 2 were taken from the soluble end of the second ethyl sulfate series. The oxides were slightly colored.

Sample 3 was taken from near the center of the second ethyl sulfate series. The oxide gave a slightly deeper color than Samples I and 2. This deeper color was due to the presence of more terbium.

2 Washburn, "Principles of Physical Chemistry," I915, p. 333. 
Samples 4,5 and 6 were obtained from the second ethyl sulfate series after 60 recrystallizations by Engle and Balke. Sample 5 was ignited at $900^{\circ}$ for 8 hours.

Sample 7 was obtained from the less soluble end of the bromate series. The oxide was slightly colored.

Sample 8 was obtained from the middle of the bromate series. The oxide was nearly white showing very little terbium to be present.

Sample 9 was also obtained from the insoluble end of the second ethyl sulfate series of Engle and Balke. After ignition for 8 hours at $850^{\circ}$ a light buff-colored oxide was obtained. When this was ignited in pure hydrogen for 3 hours at a temperature of $250^{\circ}$, the oxide became pure white. This indicated that the buff color was due to the terbium, presen: as a higher oxide. The ignition in hydrogen reduced this terbium to the trioxide $\mathrm{Tb}_{2} \mathrm{O}_{3}$. The atomic weight of this fraction was not much different from that of the others. By strong ignition in hydrogen a slate-colored oxide was formed which left a black residue upon solution in acids. When ignited at a lower temperature this slate-colored oxide was not formed. Bettendorf ${ }^{1}$ described the same phenomenon as taking place when gadolinium oxide, containing traces of terbium, was ignited in hydrogen.

All weighings were made on a Ruprecht balance, used exclusively for atomic weight work. This balance was kept in a special cork insulated room, the temperature of which remained constant to within 2 degrees. All weighings were made by the method of substitution, the tare flask being of quartz similar in size and shape to the reaction flask and differing from it in weight by only a few milligrams. The weights were carefully standardized too.o I $\mathrm{mg}$. and all weights were corrected to vacuum standard. The specific gravity of the weights was taken as 8.4 , that of dysprosium chloride as $3.67^{2}$ and that of dysprosium oxide as $7.8 \mathrm{I}^{3}$. The atomic weight of oxygen was taken as I6 and that of chlorine as 35.46. The following values were obtained by this ratio:

\begin{tabular}{|c|c|c|c|c|}
\hline Sample. & Wt. of $\mathrm{Dy}_{2} \mathrm{O}_{3}$. & Wt. of $\mathrm{DyCl}_{3}$ & $\begin{array}{c}\text { Ratio } \\
\mathrm{Dy}_{2} \mathrm{O}_{3}: \mathrm{DyCl}_{3}\end{array}$ & $\begin{array}{l}\text { Atomic } \\
\text { weight. }\end{array}$ \\
\hline $\mathbf{x} \ldots \ldots \ldots \ldots$ & 0.72974 & I. 04979 & I: 1.43859 & 163.83 \\
\hline $2, \ldots \ldots \ldots \ldots$ & 1.36017 & I. 95604 & I: 1.43808 & 164.05 \\
\hline $3 \ldots \ldots \ldots \ldots$ & 0.69716 & 1. .00255 & I: 1.43804 & 164.06 \\
\hline $4 \ldots \ldots \ldots \ldots$ & I. 02493 & I .47475 & $\mathrm{I}: \mathrm{I} \cdot \mathbf{4 3 8 8 7}$ & 163.71 \\
\hline $5 \ldots \ldots \ldots \ldots$ & 0.87688 & I. 26253 & $I: I .43979$ & $163.3 I$ \\
\hline $6 \ldots \ldots \ldots \ldots$ & 0.69318 & 0.99677 & I: I. 43796 & 163.10 \\
\hline $7 \ldots \ldots \ldots \ldots$ & I. 55248 & 2.23374 & $I: 1.43882$ & 163.72 \\
\hline $8 \ldots \ldots \ldots \ldots$ & $0.8395^{8}$ & I. 20763 & $r: 1,43837$ & 163.92 \\
\hline \multirow[t]{2}{*}{$9 \ldots \ldots \ldots \ldots$} & I. 56481 & 2.25129 & $1: 1.43869$ & 163.78 \\
\hline & & & Mean, & 163.83 \\
\hline
\end{tabular}




\section{The Ratio of Dysprosium Chloride to Silver.}

The first application of this ratio to any of the rare earths was by Baxter and Chapin ${ }^{1}$ in their determination of the atomic weight of neodymium. Baxter and Stewart ${ }^{2}$ also used this ratio in their work on the atomic weight of praseodymium. Some very concordant results were obtained by this ratio. Since anhydrous dysprosium chloride could very readily be obtained it was decided to apply the above ratio to this element also.

A preliminary determination was carried out with the use of some sodium chloride. Sodium chloride was recrystallized 3 times from conductivity water and freshly prepared hydrochloric acid. Each yield was centrifugally drained. A sample of this was fused and weighed, transferred to a glass-stoppered Jena Erlenmeyer flask of I.5 liters' capacity and dissolved in conductivity water. Silver was weighed out to within a fraction of a milligram of the calculated amount. This was dissolved in pure nitric acid in a $750 \mathrm{cc}$. Erlenmeyer flask containing a column of bulbs to retain the spray. The silver nitrate solution was diluted and added to the salt solution and the flask shaken for 2 hours in a dark room. On the following day a portion of the clear liquid was tested in the nephelometer and indicated a slight excess of chlorine. The calculated amount of silver was added from a standard solution and the flask allowed to stand for 24 hours when the nephelometer test indicated exact equivalence. In this experiment $2.96480 \mathrm{~g}$. of sodium chloride required $5.47146 \mathrm{~g}$. of silver for complete precipitation, giving 0.54186 for the ratio $\mathrm{NaCl}: \mathrm{Ag}$. Richards and Wells obtained the ratio 0.54185 .

The anhydrous dysprosium chloride, from the oxide-chloride ratio, was dissolved in the quartz flask and the solution transferred to the Erlenmeyer precipitation flask. Assuming the atomic weight of dysprosium to be I62.5, samples of silver were weighed out being usually within a milligram of the calculated amount. The weighed silver was dissolved as described above, diluted to $400 \mathrm{cc}$. and added to the dysprosium chloride of about the same dilution. The flask was then shaken for 2 or 3 hours and allowed to stand for 48 hours. The solution was then cooled to nearly $0^{\circ}$ in order to reduce the solubility of the silver chloride, and portions tested in the nephelometer. Portions of standard solutions of silver or sodium chloride were added until the nephelometer test indicated equivalence. After each addition of standard solution the flask was shaken for one hour and allowed to stand for 24 hours. In order to determine whether true equivalence was obtained the following experiments were carried out: After equivalence had been obtained in Sample I, approximately one milligram excess of silver was added and after standing for 24 hours the exact equivalent of

1 Proc. Am. Acad., 46, 2 I5 (IgIr); This Journal, 33, I (IgII); $Z$. anorg. Chem., 70, I (rgir)

2 THIS JOURNAL, 37, 516 (1915). 
sodium chloride was added. The following day exact equivalence was again found in the solution. In Sample 2 after equivalence had been obtained one milligram excess sodium chloride was added. After 24 hours its equivalent of silver was added. Equivalence was again obtained in the solution. From the foregoing tests it was proven that true equivalence was obtained in the solutions. In Sample 3 the silver ratio was not obtained because of loss in the transfer of the solution.

The weighings were carried out in the same manner as described under the oxide-chloride ratio. The specific gravity of silver was taken as I0.53 and its atomic weight as 107.88 . The following values were obtained by the chloride-silver ratio:

\begin{tabular}{|c|c|c|c|c|}
\hline Sample. & Wt. of DyCls. & Wt. of silver. & $\begin{array}{c}\text { Ratio } \\
\mathrm{DyCl}: 3 \mathrm{Ag} .\end{array}$ & $\begin{array}{l}\text { Atomic } \\
\text { weight. }\end{array}$ \\
\hline э. $\ldots \ldots \ldots \ldots$ & I. 04979 & $x .26301$ & $x: 1.20309$ & 162.62 \\
\hline $2 \ldots \ldots \ldots \ldots$ & I. 95604 & $2 \cdot 35380$ & I: 1.20334 & 162.57 \\
\hline $3 \ldots \ldots \ldots \ldots \ldots$ & I.00255 & $\ldots$ & $\ldots \ldots$ & . \\
\hline $4 \ldots \ldots \ldots \ldots$ & I. 47475 & I. 77504 & $1: 1.20362$ & $162.5 I$ \\
\hline $5 \ldots \ldots \ldots \ldots \ldots$ & 1.26253 & I. $5199 \mathrm{I}$ & I: 1.20386 & 162.45 \\
\hline $6 \ldots \ldots \ldots \ldots$ & 0.99677 & I. 20033 & I $: \mathrm{I} .2042 \mathrm{I}$ & 162.38 \\
\hline $7 \ldots \ldots \ldots \ldots$ & 2.23374 & 2.68806 & $1: 1.20338$ & 162.56 \\
\hline $8 \ldots \ldots \ldots \ldots$ & I. 20763 & I. 45325 & $1: 1.20339$ & 168.56 \\
\hline $9 \ldots \ldots \ldots \ldots$ & 2.25129 & 2.70992 & $\mathrm{I}: \mathrm{I} .2037 \mathrm{I}$ & 162.49 \\
\hline
\end{tabular}

Since the chloride-silver ratio gave values considerably lower than the oxide-chloride ratio, an attempt was made to determine whether a constant error existed in either one or both of the ratios studied. The difference in the results might be caused by one or more of the following possibilities:

I. Absorption of hydrogen chloride by the anhydrous dysprosium chloride, thus giving a lower atomic weight with the chloride-silver ratio and a higher value with the oxide-chloride ratio.

2. Volatilization of the dysprosium chloride, thus giving a higher atomic weight with the oxide-chloride ratio without affecting the result with the chloride-silver ratio.

3. Incomplete decomposition of the oxalate in igniting to the oxide. The presence of a small amount of carbonate in the oxide would give a higher atomic weight with the oxide-chloride ratio. This would not effect the chloride-silver ratio.

To determine whether hydrogen chloride was absorbed, a sample of the anhydrous chloride was prepared in the regular way and after fusion the hydrogen chloride was replaced by pure nitrogen. After all traces of hydrogen chloride had been swept out of the flask the chloride was gradually brought up to the fusion point by means of an electric furnace. A current of nitrogen was continually passed through the flask and from the outlet tube was bubbled through silver nitrate solution. As the salt began to 
fuse a gradual evolution of chloride ion was noticed. This slow evolution of chloride ion was not diminished even though the salt was kept fused for 2 hours. Upon solution of the dysprosium chloride in water a slight amount of basic chloride was formed. Since the evolution of chloride ion was not sudden at the point of fusion but began to be evolved gradually after fusion had taken place and continued to be evolved seemed to indicate that the source of the chloride ion was from the decomposition of the dysprosium chloride only.

No deposit of the chloride was noticed in the cooler outlet tube of the flask. Hopkins and Balke ${ }^{1}$ found that their anhydrous yttrium chloride did not occlude any hydrogen chloride.

Another sample of oxide was weighed and the chloride dehydrated in the regular way but not fused. The temperature was then kept at $360^{\circ}$ for 144 hours while hydrogen chloride was continually passed through the flask. At the end of this time the determination was completed in the regular way. The ratio $\mathrm{r}: \mathrm{I} .43933$ was obtained, giving an atomic weight of 163.509 . This indicated that there was no volatilization of the chloride at this temperature. Engle and Balke in their determination kept the temperature at this point for 2 hours. In the determinations as described in this paper the temperature was not held at this point for any length of time.

Engle and Balke obtained as a mean of 5 determinations the value 164.228. This value is considerably higher than that obtained in the present work. Incomplete decomposition of the oxalate in ignition to the oxide might account for this high value. The oxide in determination No. 5 of the present work was ignited considerably higher than the other samples and was found to give a much lower atomic weight by the oxidechloride ratio. Since the values obtained by the chloride-silver ratio were lower than those obtained by the oxide-chloride ratio of both the present and the former investigations, it seemed possible that in neither case had a pure oxide been obtained.

Dr. E. Wichers working in this laboratory on the oxide-chloride ratio for erbium discovered that his erbium oxide contained some carbonate. The dysprosium oxide in the present investigation was tested in a manner similar to that followed by Dr. Wichers. A sample of the oxide was ignited in the regular way for 7 hours at $800^{\circ}$ and one hour at 900 degrees. The oxide weighed $0.9299 \mathrm{gram}$. The oxide was introduced into the flask in the regular way and the flask connected to the train. Previous to this the dil. sulfuric acid in the first tower of the train had been replaced with ro\% sodium hydroxide solution and air passed through the train until no test for carbon dioxide could be obtained by passing the exit gases through Geissler bulbs containing lime water. The exit tube from the flask was 1 This Journal, 38, 2333 (1916). 
connected by means of a two-way stopcock to 2 Geissler bulbs containing freshly prepared lime water. Air was passed through for some time and the exit gases passed through one of the Geissler bulbs. No visible precipitate was obtained. The exit gas was now directed through the other Geissler bulb by turning the stopcock. While the air was passing, the inlet tube of the reaction flask was loosened and dil. nitric acid introduced. By allowing the acid to seep through the ground joint a seal was thus made and the slight back pressure in the flask prevented the air from entering. The inlet tube was now replaced and air allowed to pass for one hour. Meanwhile the flask was warmed to $60^{\circ}$ and the oxide dissolved. Quite an appreciable precipitate could be noticed in the lime water at the end of this time. From the result of this test it was proven quite conclusively that the oxide as prepared contained some carbonate. This no doubt accounts for the high result obtained by the oxide-chloride ratio.

Since platinum became too volatile and distilled into the oxide when ignited for any length of time above $900^{\circ}$ an attempt was made to ignite at a much lower temperature in high vacuo. Accordingly a platinum boat containing the oxalate was placed in a quartz tube and ignited for 8 hours at $700^{\circ}$ by means of an electric tube furnace. During this ignition the quartz tube was kept evacuated to less than I mm. pressure. The tube was then allowed to cool and dry carbon-dioxide-free air was allowed to enter until atmospheric pressure had been reached. The transfer of the oxide to the reaction flask was made in an air-tight box. This box was made of wood well impregnated with paraffin and contained a sliding plate glass cover. The reaction flask was placed in the box and the end of the quartz combustion tube was fitted into a hole cut in one end of the box. Dry carbon-dioxide-free air was passed into the box for some time and in addition an open dish of solid potassium hydroxide was placed inside to absorb the carbon dioxide. By inserting the hands into rubber gloves fitted into holes cut in the sides of the box the boat could be drawn from the tube and the transfer made with the minimum amount of carbon dioxide present. The value obtained by the oxide-chloride ratio in this case was 167.46 as the atomic weight. Two more determinations were carried out by this modification. In place of the platinum boat an alundum boat of the highest grade alundum was used and a temperature of approximately $950^{\circ}$ was maintained in the ignition. The values 164.26 and 167.61 were obtained.

One more modification was tried. Two samples of the oxalate were ignited in an alundum crucible for 10 hours at a temperature of $1000^{\circ}$ and the transfer was made in the regular way as described in the former part of this paper. The values obtained were 164.55 and 164.40 .

It seems probable that the oxide when allowed to cool absorbs carbon dioxide with extreme rapidity and does not lend itself to the manipulation 
as here used. It also appears that the hot oxide when transferred in the air absorbs enough carbon dioxide to vitiate the results. From the experimental data obtained it seems quite evident that the oxide-chloride ratio cannot be applied to dysprosium by the methods thus far tried. The ratio is accordingly being abandoned in favor of the chloride-silver ratio which has given good results in this laboratory.

\section{Io. Summary.}

1. Dysprosium material already in a high state of purity was further fractionated as the ethyl sulfates and the bromates. The ethyl sulfates were found to be more efficient for the separation of dysprosium from neodymium, praseodymium and terbium. Neither of the two methods was found efficient for the separation of dysprosium from holmium.

2. The hydrate $\mathrm{Dy}_{2}\left(\mathrm{SO}_{4}\right)_{3} \cdot 8 \mathrm{H}_{2} \mathrm{O}$ was not found to be constant in composition when dried over sulfuric acid.

3. The ratio $\mathrm{Dy}_{2} \mathrm{O}_{3}: \mathrm{DyCl}_{3}$ was further studied. The value $\mathrm{I}_{6} \mathrm{H}_{3} .83$ as the atomic weight was obtained as the mean of 9 determinations. A study has been made to account for the difference of this value and that of a higher value ( 164.228 ) obtained in a previous investigation.

4. In studying the ratio $\mathrm{DyCl}_{3}: 3 \mathrm{Ag}$ the value $162.5^{2}$ was obtained as the mean of 8 determinations. A comparative study of the two values has been made to account for the difference in the values obtained by the two methods.

5. The dysprosium oxide as prepared for the oxide-chloride ratio by ignition of the oxalate was found to contain some carbonate. Several different methods for preparing a pure oxide were tried but by no method could concordant results be obtained by the oxide-chloride ratio.

6 . The results obtained by the oxide-chloride ratio are accordingly discarded in favor of the results ( 162.52 ) obtained by the chloride-silver ratio.

URBANA, ILL.

\section{THE LEAD ELECTRODE. [SECOND COMMUNICATION.] \\ BX FREDERTCK H. GatMAN. \\ Received January 28, 1918.}

Since the publication of a previous paper ${ }^{1}$ on the lead electrode some doubt has arisen as to whether lead really exhibits the phenomenon of allotropy. ${ }^{2}$ If the metal does exist in more than one form, each modification should possess its own specific potential, and a careful study of the electromotive behavior of lead electrodes prepared in various different ways should afford valuable evidence as to whether or not allotropes exist.

1 Getman, This Journal, 38, 792 (I9r6).

2 Lewis and Brighton, Ibid., 39, 1906 (I917). 